\title{
Cryopreservation of protocorm-like bodies (PLBs) of Phalaenopsis bellina (Rchb.f.) christenson by encapsulation-dehydration
}

\begin{abstract}
Protocorm-like bodies (PLBs) of Phalaenopsis bellina were successfully cryopreserved by the encapsulation-dehydration approach. Various stages in obtaining successful cryopreservation using this method were optimized. Encapsulated PLBs precultured in half-strength MS medium supplemented with $0.75 \mathrm{M}$ sucrose for 3 days exhibited the highest viability in terms of 2,3,5-triphenyltetrazoliumchloride (TTC) reduction. The amount of sucrose in the PLBs after incubation in different concentrations of sucrose for different periods of time determined by HPLC. The highest sucrose concentration was $7 \mathrm{mg} / \mathrm{g}$ of PLBs for the PLBs treated with $0.75 \mathrm{M}$ sucrose for 3 days as compared to the control which had only $1 \mathrm{mg} / \mathrm{g}$ sucrose. After sucrose preculture, the PLBs were subjected to desiccation using one of two methods. Desiccation using silica gel was more efficient in reducing PLBs moisture content. After $6 \mathrm{~h}$ of desiccation, PLBs desiccated using laminar air flow had $43.5 \%$ moisture content while for those desiccated using silica gel had $32 \%$ moisture content. PLBs desiccated to different moisture contents were plunged into LN. After storage in LN the encapsulated PLBs were rewarmed. Two weeks after re-warming PLBs viability was determined by TTC reduction and re-growth assessed. Encapsulated PLBs precultured with $0.75 \mathrm{M}$ sucrose for 3 days followed by desiccated using silica gel for $5 \mathrm{~h}$ resulting in a moisture content of $39 \%$ lead to the highest post re-warming viability in terms of TTC reduction (46.6\% of control PLBs) and $30 \%$ re-growth.
\end{abstract}

Keyword: Moth orchid; Germplasm conservation; TTC; Sodium alginate HPLC 